\title{
MATRICES WITH PRESCRIBED ENTRIES AND CHARACTERISTIC POLYNOMIAL
}

\author{
J. A. DIAS da SILVA
}

ABSTRACT. It is proved that there exists an $n \times n$ matrix over an arbitrary field with $n-1$ prescribed entries and prescribed characteristic polynomial.

In [3] the author proves that there always exists an $n \times n$ matrix over an arbitrary field $\phi$, with $n-1$ prescribed entries and prescribed eigenvalues in $\phi$.

Answering a question posed at the end of G. N. de Oliveira's paper, we shall solve the problem of finding a necessary and sufficient condition for the existence of an $n \times n$ matrix over $\phi$, with $n-1$ prescribed entries and prescribed characteristic polynomial.

Our principal results are the following:

Theorem 1. Let $a_{1}, \cdots, a_{n-1} \in \phi$, and $f(\lambda)$ be a monic polynomial of degree $n$ over $\phi$. Let $\left(i_{1}, j_{1}\right), \cdots,\left(i_{n-1}, j_{n-1}\right)$ be prescribed distinct positions in an $n \times n$ matrix. Suppose the following condition is not satisfied:

(1) The prescribed positions are all the nonprincipal positions of a row or column and $a_{1}=\cdots=a_{n-1}=0$.

Then there exists an $n \times n$ matrix over $\phi$ with $a_{t}$ in the position $\left(i_{t}, j_{t}\right)$, $t=1, \cdots, n-1$, and with $f(\lambda)$ as characteristic polynomial.

Theorem 2. With the same notation as in Theorem 1 ass ume that the condition (1) is satisfied. Then there exists an $n \times n$ matrix over $\phi$ with $a_{t}$ in the position $\left(i_{t}, j_{t}\right), t=1, \cdots, n-1$, and $f(\lambda)$ as characteristic polynomial if and only if $f(\lambda)$ has at least one root in $\phi$.

Before bringing out the proofs of these theorems we will prove some auxiliary results.

Let $\phi_{n}$ be the vector space over $\phi$ of the $n \times 1$ matrices over $\phi$. If $v \in \phi_{n}$ and $B$ is an $n \times n$ matrix over $\phi$, the minimal polynomial of $v$ relative

Received by the editors November 17, 1972 and, in revised form, June 19, 1973. AMS (MOS) subject classifications (1970). Primary 15A15; Secondary 15A18. 
to $B$ is the monic polynomial $g(\lambda)$ over $\phi$ of least degree, such that $g(B) v=$ 0 . We say that $v$ has property $\beta$ relative to $B$, if the minimal polynomial of $v$ relative to $B$ is equal to the characteristic polynomial of $B$.

All matrices and polynomials considered in the present paper are over the arbitrary field $\phi$.

Lemma 1. Let $B$ be an $(n-1) \times(n-1)$ matrix and $f(\lambda)$ a monic polynomial of degree $n$.

If $v \in \phi_{n-1}$ has property $\beta$ relative to $B$, then it is possible to border below the $(n-1) \times n$ matrix $[B \quad v]$ with a row to obtain an $n \times n$ matrix with characteristic polynomial $f(\lambda)$.

Proof. This result is essentially in [1]. Since it is not explicitly stated in [1], we shall present here a short proof.

Since $v$ has property $\beta$ relative to $B, v, B v, \cdots, B^{n-2} v$ form a bas is for $\phi_{n-1}$. Let $\phi_{n-1}^{*}$ be the dual space of $\phi_{n-1}$. Let $g \in \phi_{n-1}^{*}$. Define

$$
\theta: \phi_{n-1}^{*} \rightarrow \phi_{n-1}, \quad g \rightarrow \theta(g)=\left(g(v), g(B v), \cdots, g\left(B^{n-2} v\right)\right) .
$$

It is well known that $\theta$ is an isomorphism. Since $g$ may be represented by an $(n-1) \times 1$ column $Q$, it follows that while $Q$ runs over $\phi_{n-1}, \theta(g)=$ $\left(Q^{T} v, Q^{T} B v, \cdots, Q^{T} B^{n-2} v\right)$ (the superscript $T$ means transpose) runs over $\phi_{n-1}$. Let

$$
A=\left[\begin{array}{ll}
B & v \\
Q^{T} & b
\end{array}\right]
$$

We show that we can choose $Q \in \phi_{n-1}$ and $b \in \phi$ so that the characteristic polynomial of $A$ is $f(\lambda)$. In [1] the authors proved that

$$
\operatorname{det}\left(\lambda I_{n}-A\right)=\lambda^{n}+\left(t_{1}-b\right) \lambda^{n-1}+h(\lambda)-\sum_{r=0}^{n-2} U_{r}(\lambda) Q^{T} B^{r} v
$$

where $I_{n}$ is the $n \times n$ identity matrix, $-t_{1}$ is the trace of $B, b(\lambda)$ is a polynomial of degree at most $n-2$ and $U_{0}(\lambda), \cdots, U_{n-2}(\lambda)$ are certain polynomials which form a basis for the space of all polynomials over $\phi$ of degree at most $n-2$. From this property of the $U_{i}(\lambda)$ and from the fact that $\left(Q^{T} v, Q^{T} B v, \cdots, Q^{T} B^{n-2} v\right)$ runs over $\phi_{n-1}$ as $Q$ runs over $\phi_{n-1}$, it follows that we can choose $Q$ so that

$$
h(\lambda)-\sum_{r=0}^{n-2} U_{r}(\lambda) Q^{T} B^{r} v
$$

is equal to any prescribed polynomial of degree at most $n-2$. Choosing an 
appropriate value for $b$ it is clear that we can make $\operatorname{det}\left(\lambda I_{n}-A\right)=f(\lambda)$.

Lemma 2. Let $B$ be an $n \times n$ matrix with irreducible characteristic polynomial. If $v$ is an arbitrary nonzero vector of $\phi_{n}$, then $v$ has property $\beta$ relative to $B$.

Proof. It is clear, since the minimal polynomial of $v$ relative to $B$ must be a divisor of the characteristic polynomial of $B$.

Lemma 3. Let $B$ be an $n \times n$ matrix $(n>2)$ over $\phi$ with $n$ distinct characteristic roots in $\phi$. We assume that $\phi$ is not the trivial field consisting only of 0 and 1. Let a be a nonzero element of $\phi$ and $i$ a fixed positive integer smaller than $n+1$. Then there exists a vector $v \in \phi_{n}$ with property $\beta$ relative to $B$ and with ith coordinate equal to $a$.

Proof. Let $v_{i}$ be the characteristic vector of $B$ corresponding to the characteristic root $\lambda_{i}$. The minimal polynomial of $v_{i}$ is $\lambda-\lambda_{i}$, and since $\lambda_{i} \neq \lambda_{j}$ for $i \neq j$, these minimal polynomials are pairwise prime. Therefore the minimal polynomial of the vector $v=\sum_{j=1}^{n} \alpha_{j} v_{j}$, where the $a_{j}$ are arbitrary nonzero elements of $\phi$, is the characteristic polynomial of $B$. Of course we can choose the $a_{j}$ so that the $i$ th coordinate of $v$ is $a$.

Lemma 4. Under the same hypothesis as in Lemma 3 let a $=0$. Suppose that at least one of the nonprincipal elements of the ith row of $B$ is not zero. Then there exists a vector $v \in \phi_{n}$ with property $\beta$ relative to $B$ and with ith coordinate equal to 0 .

Proof. Let $v_{i}$ be the characteristic vector of $B$ corresponding to the characteristic root $\lambda_{i}$. If at least two of the $v_{j}$ have $i$ th coordinate different from zero, it is clear that we can choose the $\alpha_{j}$ appearing in the proof of Lemma 3 so that the ith coordinate of $v$ be zero. To complete the proof we show that the assumption that $n-1$ of the $v_{j}$ have $i$ th coordinate equal to zero leads to contradiction. Let $v_{j}=\left(v_{j}^{1}, \cdots, v_{j}^{n}\right)$. Assume that $v_{1}^{i}=\cdots=$ $v_{b-1}^{i}=v_{b+1}^{i}=\cdots=v_{n}^{i}=0$. Since the $v_{k}$ are characteristic vectors of $B$ we have

$$
\sum_{j=1 ; j \neq i}^{n} b_{i j} v_{k}^{j}=0, \quad k=1, \cdots, h-1, h+1, \cdots, n .
$$

By assumption at least one of $b_{i 1}, \cdots, b_{i-1}, b_{i+1}, \cdots, b_{i n}$ is different 
from zero. The above inequality means that the matrix $\left[v_{k}^{j}\right], j=1, \cdots, i-$ $1, i+1, \cdots, n ; k=1, \cdots, b-1, b+1, \cdots, n$ is singular, i.e., that the vectors $v_{1}, \cdots, v_{b-1}, v_{b+1}, \cdots, v_{n}$ are linearly dependent. This is a contradiction.

Lemma 5. Let $\phi$ be infinite and $a_{1}, \cdots, a_{n} \in \phi$. Assume that $\left(i_{1}, j_{1}\right)$, $\cdots,\left(i_{n}, j_{n}\right)$ are prescribed positions of an $n \times n$ matrix not all on the same diagonal. Then there exists an $n \times n$ matrix with $a_{t}$ in the position $\left(i_{t}, j_{t}\right), t=1, \cdots, n$, and distinct characteristic roots.

Proof. It is an immediate consequence of results proved in [4].

Proof of Theorem 1. There is [2] an integer $s, 1 \leq s \leq n$, such that either (1) there is exactly one prescribed entry in the $s$ th row or in the $s$ th column, but not both, or (2) there are no prescribed entries in the $s$ th row and sth column. Performing a permutation on the rows of the matrix and the same permutation on its columns we may assume, without loss of generality, that $s=n$. Transposing the matrix, if necessary, we can also assume that in Case (1) it is the $n$th column that has a prescribed entry.

Case (1). We have to consider two subcases.

Subcase A. The field $\phi$ is finite. The proof is by induction on the order of the matrix. For $n=2$ the theorem is trivially true. Let $\left(i_{n-1}, j_{n-1}\right)$ denote the prescribed entry of the $n$th column $(n>2)$. Assume that not all of the positions $\left(i_{1}, j_{1}\right), \cdots,\left(i_{n-2}, j_{n-2}\right)$, are nonprincipal positions of one row or column or if they are, that at least one of $a_{1}, \cdots, a_{n-2}$ is not zero. Let $g(\lambda)$ be an irreducible polynomial of degree $n-1$ (over a finite field there are irreducible polynomials of every degree [5, p. 506]). By induction there exists an $(n-1) \times(n-1)$ matrix $B$ with characteristic polynomial $g(\lambda)$ and $a_{1}, \cdots, a_{n-2}$ in the positions $\left(i_{1}, j_{1}\right), \cdots,\left(i_{n-2}, j_{n-2}\right)$, respectively. Let $v$ be a nonzero vector of $\phi_{n-1}$ with $i_{n-1}$ th coordinate equal to $a_{n-1}$. By Lemmas 2 and 1 it is possible to border below [B $v$ ] with a row to obtain a matrix $A$ with characteristic polynomial $f(\lambda) . A$ is the required matrix. Assume now that $n>3$ and $\left(i_{1}, j_{1}\right), \cdots,\left(i_{n-2}, j_{n-2}\right)$ are nonprincipal positions of a row (for a column the problem is solved in the same way) and $a_{1}=\cdots=a_{n-2}=0$. By the assumption of the theorem, if $i_{n-1}$ is equal to the common value of $i_{1}, \cdots, i_{n-2}$, then $a_{n-1} \neq 0$. It can be easily seen that we can reduce the problem to the previous case by interchanging the rows $n$ and $k$ (for any $k$ different from $n, i_{n-1}$ and $i_{n-2}$ ) and the columns $n$ and $k$. The case $n=3$ needs a special study. In this case if one of the two prescribed entries is different from zero applying an 
appropriate permutation of the rows and columns and transposition if necessary we can overcome the difficulty. Suppose both prescribed entries are zero. Permuting rows and columns if necessary, we may assume that the prescribed entries are $(1,2)$ and $(2,3)$. Take

$$
B=\left[\begin{array}{ll}
0 & 0 \\
1 & 1
\end{array}\right] \text { and } v=\left[\begin{array}{l}
1 \\
0
\end{array}\right] \text {. }
$$

It can be easily seen that $v$ has property $\beta$ relative to $B$. Applying Lemma 1 to $B$ and $v$ we see that there is a $3 \times 3$ matrix $A$ with $a_{12}=a_{23}=0$ and prescribed characteristic polynomial.

Subcase B. The field $\phi$ is infinite. Suppose $a_{n-1} \neq 0$. By Lemma 5 we can construct an $(n-1) \times(n-1)$ matrix $B$ with $a_{t}$ in the position $\left(i_{t}, j_{t}\right)$, $t=1, \cdots, n-2$, and distinct characteristic roots. Now Lemmas 3 and 1 solve our problem. Suppose $a_{n-1}=0$. By the hypothesis either all the nonprincipal elements of the $i_{n-1}$ th row are prescribed, one of them being different from zero, or at least one of the nonprincipal positions of the $i_{n-1}$ th row, (the $\left(i_{n-1}, q\right)$ position, say), is not prescribed. In this case we prescribed to the $\left(i_{n-1}, q\right)$ position an arbitrary nonzero value. We see that, in either case, Lemma 5 enables us to construct an $(n-1) \times(n-1)$ matrix with distinct characteristic roots, $a_{t}$ in the position $\left(i_{t}, j_{t}\right), t=1, \cdots, n-2$ and zero element in the $i_{n-1}$ th row. Now Lemmas 4 and 1 solve the problem.

Case (2). We also have to consider two subcases.

Subcase A. The field $\phi$ is finite. The proof is by induction. For $n=2$ the problem is easily solved. Suppose now that not all of $\left(i_{1}, j_{1}\right), \cdots$, $\left(i_{n-2}, j_{n-2}\right)$ are nonprincipal positions of a row or column or, if they are, that at least one of $a_{1}, \cdots, a_{n-2}$ is not zero. By the induction hypothes is there exists an $(n-1) \times(n-1)$ matrix $B$ with $a_{t}$ in position $\left(i_{t}, j_{t}\right), t=1$, $\cdots, n-2$, and irreducible characteristic polynomial. Take

$$
v=\left[\begin{array}{llllllll}
0 & \cdots & 0 & b & 0 & \cdots & 0
\end{array}\right]^{T}(n-1) \text { rows, }
$$

where $b$ is in the $i_{n-1}$ th row and is an arbitrary nonzero element of $\phi$. By Lemmas 2 and 1 there is an $n \times n$ matrix $A$ whose submatrix contained in the first $n-1$ rows is equal to $[B \quad v]$ and with characteristic polynomial $f(\lambda)$. If $a_{i_{n-1}, j_{n-1}}=a_{n-1}, A$ is the required matrix. If $a_{i_{n-1}, j_{n-1}} \neq a_{n-1}$ take $b=a_{i_{n-1}, j_{n-1}}-a_{n-1}$. After constructing $A$ add its $j_{n-1}$ th row to the last and subtract the $n$th column from the $j_{n-1}$ th column (a similarity transformation). The matrix so obtained satisfies the requirements of the theorem. 
If $\left(i_{1}, j_{1}\right), \cdots,\left(i_{n-2}, j_{n-2}\right)$, are the nonprincipal positions of a row (or column), $a_{1}=\cdots=a_{n-2}=0$ and $n>3$, we interchange the rows $n$ and $k$ (where $k$ is different from $n, i_{n-1}$ and $i_{n-2}$ ) and the columns $n$ and $k$. Thus we reduce the problem to the case just treated. For $n=3$ a difficulty arises only if $\left(i_{1}, j_{1}\right)=(1,2),\left(i_{2}, j_{2}\right)=(2,1)$ and $a_{1}=a_{2}=0$. In this case take

$$
B=\left[\begin{array}{ll}
0 & 0 \\
0 & 1
\end{array}\right] \quad \text { and } \quad v=\left[\begin{array}{l}
1 \\
1
\end{array}\right]
$$

The vector $v$ has property $\beta$ relative to $B$. Applying Lemma 1 to $B$ and $v$ we see that there is a solution.

Subcase $B$. The field $\phi$ is infinite. If the prescribed entries are not on a diagonal it is possible by Lemma 5 to construct an $(n-1) \times(n-1)$ matrix $B$ with $a_{t}$ in the position $\left(i_{t}, j_{t}\right), t=1, \cdots, n-1$, and distinct characteristic roots. By Lemma 3 there exists a nonzero $(n-1) \times 1$ vector $v$ with property $\beta$ relative to $B$. By Lemma 1 there is an $n \times n$ matrix satisfying the requirements of our problem.

Now, applying Lemmas 3 and 1 , there is no difficulty in seeing that there exists an $n \times n$ matrix satisfying the requirements of our problem. If the prescribed entries are principal entries it is well known that our problem has a solution. Suppose now that the prescribed entries are on a nonprincipal diagonal. By Lemma 5 there exists an $(n-1) \times(n-1)$ matrix $B$ with $a_{t}$ in the position $\left(i_{t}, j_{t}\right), t=1,2, \cdots, n-2$, and distinct characteristic roots. Suppose $b_{i_{n-1},{ }_{n-1}} \neq a_{n-1}$. By Lemma 3 there exists an $(n-1) \times 1$ column $v$ such that $v$ has property $\beta$ relative to $B$ and its $i_{n-1}$ th coordinate is equal to $b_{i_{n-1}, j_{n-1}}-a_{n-1}$. There exists by Lemma 1 an $n \times n$ matrix $A$ with $f(\lambda)$ as characteristic polynomial and whose $(n-1) \times n$ matrix contained in its first $n-1$ rows is $\left[\begin{array}{ll}B & v\end{array}\right]$. Now add the $j_{n-1}$ th row of $A$ to its last row and then subtract its last column from its $j_{n-1}$ th column. We get a matrix that satisfies the conditions of our problem. The proof of Theorem 1 is complete. The orem 2 is an easy consequence of The orem 1 .

Acknowledgment. I wish to thank the refree for pointing out an error in a previous version of Lemma 3. I also wish to thank G. N. Olive ira and Alice Inácio for the ir helpful remarks.

\section{REFERENCES}

1. H. K. Farahat and W. Ledermann, Matrices with prescribed characteristic polynomials, Proc. Edinburgh Math. Soc. 11 (1958/59), 143-146. MR 21 \#6382. 
2. D. London and H. Minc, Eigenvalues of matrices with prescribed entries, Proc. Amer. Math. Soc. 34 (1972), 8-14.

3. G. N. de Oliveira, Matrices with prescribed entries and eigenvalues. I, Proc. Amer. Math. Soc. 37 (1973), 380-386.

4. - Matrices with prescribed entries and eigenvalues. II, Siam J. Appl. Math. 24 (1973), 414-417.

5. L. Rédei, Algebra. Vol. 1, Akad, Kiadó, Budapest, 1954; English transl., Pergamon Press, New York, 1967. MR 16, 559; 35 \#2697.

INSTITUTO DE F ÍSICA E MATEMÁTICA, 2, AV. GAMA PINTO, LISBOA-4, PORTUGAL 